\title{
Cytotoxic Effects of Jay Amin Hydroxamic Acid (JAHA), a Ferrocene- Based Class I Histone Deacetylase Inhibitor, on Triple-Negative MDA- MB231 Breast Cancer Cells
}

\author{
Mariangela Librizzi, ${ }^{\dagger}$ Alessandra Longo, ${ }^{\dagger}$ Roberto Chiarelli, ${ }^{\dagger}$ Jahanghir Amin, ${ }^{\ddagger}$ John Spencer, ${ }^{\S}$ \\ and Claudio Luparello*,† \\ †Dipartimento STEMBIO, Edificio 16, Università di Palermo, Viale delle Scienze, 90128 Palermo, Italy \\ ${ }^{\ddagger}$ School of Science at Medway, University of Greenwich, Kent ME4 4TB, United Kingdom \\ ${ }^{\S}$ Department of Chemistry, School of Life Sciences, University of Sussex, Falmer, Brighton BN1 9QJ, United Kingdom
}

ABSTRACT: The histone deacetylase inhibitors (HDACis) are a class of chemically heterogeneous anticancer agents of which suberoylanilide hydroxamic acid (SAHA) is a prototypical member. SAHA derivatives may be obtained by three-dimensional manipulation of SAHA aryl cap, such as the incorporation of a ferrocene unit like that present in Jay Amin hydroxamic acid (JAHA) and homo-JAHA [Spencer, et al. (2011) ACS Med. Chem. Lett. 2, 358-362]. These metal-based SAHA analogues have been tested for their cytotoxic activity toward triple-negative MDAMB231 breast cancer cells. The results obtained indicate that of the two compounds tested, only JAHA was prominently active on breast cancer cells with an $\mathrm{IC}_{50}$ of $8.45 \mu \mathrm{M}$ at $72 \mathrm{~h}$ of treatment. Biological assays showed that exposure of MDA-MB231 cells to the HDACi resulted in cell cycle perturbation with an alteration of $S$ phase entry and a delay at $\mathrm{G}_{2} / \mathrm{M}$

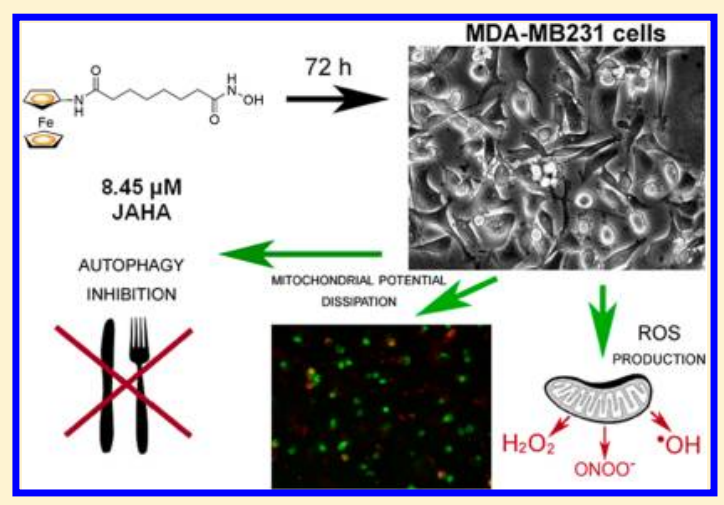
transition and in an early reactive oxygen species production followed by mitochondrial membrane potential (MMP) dissipation and autophagy inhibition. No annexin binding was observed after short- $(5 \mathrm{~h})$ and longer $(24$ and $48 \mathrm{~h})$ term incubation with JAHA, thereby excluding the promotion of apoptosis by the HDACi. Although caution must be exercised in extrapolation of in vitro results to the in vivo situation for which research on animals and human trials are needed, nevertheless JAHA treatment possesses the potential for its development as an agent for prevention and/or therapy of "aggressive" breast carcinoma, thus prompting us to get more insight into the molecular basis of its antibreast cancer activity.

\section{INTRODUCTION}

Histones are a group of highly alkaline proteins that bind DNA and pack it in the characteristic nucleosomal architecture of chromatin in the eukaryotic cell nuclei. Post-translational modifications of histones are known to promote subtle, precisely regulated alterations of chromatin organization, thereby modulating the chromatin-mediated processes occurring in the nucleus, such as transcription, replication, and repair. ${ }^{1}$ Among the enzymes involved in chromatin remodelling, histone deacetylases (HDACs) catalyze the deacetylation from lysine residues in the $\mathrm{N}$-terminal ends of nucleosomal histones, leading to chromatin condensation, thus controlling the transcriptional activity of numerous genes often associated with the onset of human diseases, such as inflammation, cancer, and neurodegeneration. ${ }^{2-4}$ In addition, HDACs also play key regulatory roles via interactions with nuclear receptors and transcription factors, such as the retinoblastoma protein and Max-Mad, ${ }^{5,6}$ and also control cytoskeleton architecture and nuclear import via acetylation of tubulin and importin $\alpha{ }^{7}$ thereby exerting multifold nonhistone targeted effects in the intracellular microenvironment. Four classes of HDACs have been recognized, of which class I comprises four members
(HDAC1, $-2,-3$, and -8 ) that are found almost exclusively in the cell nucleus. ${ }^{8}$

The histone deacetylase inhibitors (HDACis) are an emerging class of chemically heterogeneous anticancer agents, which restore a relaxed, hyperacetylated, chromatin structure potentially leading to re-expression of silenced genes and/or silencing of downstream genes, ultimately inducing death, apoptosis, and cell cycle arrest in cancer cells also via nonhistone-targeted mechanisms such as perturbations of p53, cytokine signaling pathways, and angiogenesis. ${ }^{9,10}$ HDACis are grouped in different categories on the basis of their chemical nature, and the implication of this molecular diversity is that the precise mechanism through which these compounds work is still poorly understood. Suberoylanilide hydroxamic acid (SAHA), a.k.a. Vorinostat, is a prototypical HDACi targeting class I and II HDAC approved in 2006 by the Food and Drug Administration for treatment of cutaneous Tcell lymphoma, ${ }^{11}$ which has been subjected to several manipulations of the core structure to probe the effect of

Received: September 5, 2012 
such modifications on both HDAC inhibitory activity and cytotoxic effect on cancer cells. Some of us ${ }^{12}$ have recently reported the synthesis of metal-based SAHA analogues incorporating a ferrocene unit, that is, Jay Amin hydroxamic acid (JAHA a.k.a. JA47) and homo-JAHA, which, if compared with parental SAHA, displayed similar broad inhibitory profiles toward class I HDACs, including HDAC8, although homoJAHA was less potent toward HDAC6 and more effective toward HDAC8, whereas none of them was active on class IIa HDACs. The ability of these compounds to restrain the growth of the estrogen receptor-positive, low malignant, MCF-7 breast tumor cells prompted us to extend the investigation to other neoplastic cell lines, more representative of breast cancer histotypes with poor outcome, and also to get more insight into the molecular mechanism of cytotoxic induction.

Triple-negative breast cancers lack expression of estrogen and progesterone receptors and are human epidermal growth factor receptor 2 (HER2)/neu negative, thereby being poorly responsive to hormonal therapies and to HER2-targeting drugs and usually associated with worse prognosis than other breast tumor types. ${ }^{13}$ The MDA-MB231 cell line has been chosen as the model system for this study, being derived from a pleural effusion of a triple-negative breast cancer of basal subtype and displaying several features of an aggressive phenotype, such as the presence of an inactive mutant form of $\mathrm{p} 53$, and the invasiveness and formation of metastasis in vivo. ${ }^{44,15}$ Here, we report that JAHA is more efficient than homo-JAHA as an anticancer agent toward MDA-MB231 cells, thus being worthy of further in vivo investigations, and that its cytotoxic activity, when administered at $72 \mathrm{~h}$ half maximal inhibitory concentration $\left(\mathrm{IC}_{50}\right)$ concentration, sees the involvement of (i) cell cycle perturbation, possibly related to an inability of cells to proceed through the $\mathrm{G}_{2} / \mathrm{M}$ transition, (ii) an early increase of reactive oxygen species (ROS) production followed by dissipation of mitochondrial transmembrane potential, and (iii) the inhibition of the autophagic process.

\section{EXPERIMENTAL PROCEDURES}

Cell Culture and HDACis. MDA-MB231 breast tumor cells were maintained in RPMI 1640 medium plus $10 \%$ fetal calf serum (FCS), $100 \mathrm{U} / \mathrm{mL}$ penicillin, $100 \mu \mathrm{g} / \mathrm{mL}$ streptomycin, and $2.5 \mathrm{mg} / \mathrm{L}$ amphotericin B (Invitrogen, Carlsbad, CA), at $37{ }^{\circ} \mathrm{C}$ in a $5 \% \mathrm{CO}_{2}$ atmosphere. The cells were detached from flasks with $0.05 \%$ trypsinEDTA, counted, and plated at the necessary density for treatment after achieving $60-80 \%$ confluency. JAHA and homo-JAHA were synthesized as reported by Spencer et al. ${ }^{12}$ and dissolved at $6.5 \mathrm{mM}$ concentration in dimethyl sulfoxide (DMSO) as stock solutions.

Cell Viability Assay. The assessment of in vitro cytotoxic activity was determined by an 3-(4,5-dimethylthiazol-2-yl)-2,5-diphenyltetrazolium bromide (MTT) assay (http://www.molmeth.org/protocols/ 1VB7RPF). Briefly, MDA-MB231 cells in exponential growth were plated at a concentration of 5500 cells/well in a 96-well plate and allowed to adhere overnight. Then, the cells were treated with $0.1,1$, 10 , and $100 \mu \mathrm{M}$ concentration of either drug or with the vehicle (DMSO) for 24, 48, and $72 \mathrm{~h}$ and observed under the phase-contrast microscope before processing for the viability assay. Different amounts of DMSO, equivalent to those diluting the drugs in the parallel experiments, were added to cells in the conditions of vehicle-only treatment. Fifteen microliters of MTT (final concentration, $0.75 \mathrm{mg}$ / $\mathrm{mL}$ ) was then added, and after incubation, $100 \mu \mathrm{L}$ of SDS-containing solubilization buffer replaced the MTT-containing culture medium and dissolved the precipitated violet formazan crystals within metabolically viable cells. Absorbance of the dissolved dye was measured in an automated microplate reader at $550 \mathrm{~nm}$. The cell viability ratio was determined as the ratio between treated cells and untreated controls. $\mathrm{IC}_{50}$ was calculated with Prism 5.0 software (GraphPad, La Jolla, CA).

Apoptosis Assay. The possible onset of apoptosis was evaluated using the Annexin V-fluorescein isothiocyanate (FITC) kit (Miltenyi Biotec $\mathrm{GmbH}$, Bergisch Gladbach, Germany) according to manufacturer's instructions. Briefly, plated cells were treated with $8.45 \mu \mathrm{m}$ JAHA for 5, 24, and $48 \mathrm{~h}$. Then, attached and floating cells were collected and resuspended in $100 \mu \mathrm{L}$ of binding buffer $1 \times / 10^{6}$ cells, and $10 \mu \mathrm{L}$ of FITC-annexin V was added. After centrifugation and resuspension in $500 \mu \mathrm{L}$ of binding buffer $1 \times$, propidium iodide was added immediately prior to flow cytometric analysis in a FACSCanto apparatus (BD Biosciences, Franklin Lakes, NJ). Data were represented as dot plots using Flowing software v.1.6.0., which discriminated normal cells (bottom left quadrant) from cells in early apoptosis (bottom right quadrant), cells in late apoptosis or early necrosis (top right quadrant), or cells undergoing necrosis (top left quadrant). Vehicle-treated cells were tested as parallel controls.

Cell Cycle Analysis. MDA-MB231 cells were seeded in six-well plates at a concentration of 88000 cells/well and allowed to adhere overnight and then treated with $8.45 \mu \mathrm{m}$ JAHA and harvested after 24 and $48 \mathrm{~h}$ of incubation. Cells were resuspended in $0.5 \%$ Triton X-100 in phosphate-buffered saline containing $0.5 \mu \mathrm{g} / \mathrm{mL}$ RNase A, incubated a $37{ }^{\circ} \mathrm{C}$ for $30 \mathrm{~min}$, and then treated with propidium iodide at $4{ }^{\circ} \mathrm{C}$ for $30 \mathrm{~min}$ in the dark. The cell cycle distribution was analyzed by flow cytometry in a FACSCanto apparatus and Weasel 3.0.1 software. Vehicle-treated cells were tested as parallel controls.

Evaluation of ROS Production. The production of ROS, such as hydrogen peroxide, peroxynitrite, hydroxyl radicals, nitric oxide, and peroxy radical, and of superoxide was checked using the Total ROS/ Superoxide Detection Kit (Enzo Life Sciences, Lausen, Switzerland) according to the manufacturer's instructions. Briefly, attached and floating MDA-MB231 cells treated with $8.45 \mu \mathrm{m}$ JAHA were harvested after 24 and $48 \mathrm{~h}$ of incubation and resuspended in $500 \mu \mathrm{L}$ of ROS/ Superoxide Detection Mix and incubated for $30 \mathrm{~min}$ at $37{ }^{\circ} \mathrm{C}$ in the dark prior to flow cytometry assay for the green and orange fluorescence in a FACSCanto apparatus. Vehicle-treated cells were tested as parallel controls. Positive (pyocyanin-treated) and negative ( $N$-acetyl-L-cysteine-treated) controls were included in the analysis, and data were represented as dot plots using Flowing software v.1.6.0., which discriminate cells with increased total ROS production (top left quadrant), increased superoxide production (bottom right quadrant), and increased total ROS and superoxide production (top right quadrant). To assess the involvement of ROS production in the cytotoxic effect of JAHA, an MTT assay was performed as already reported on JAHA-treated cells incubated for $24 \mathrm{~h}$ in the presence of the antioxidant butylated hydroxytoluene $(\mathrm{BHT})^{16}$ at $5,10,25$, and 50 $\mu \mathrm{m}$ final concentration.

Mitochondrial Membrane Potential (MMP) Assay. Control and JAHA-treated cell preparations were stained with the fluorescent dye JC1 (Invitrogen) according to Cannino et al., ${ }^{17}$ and MMP was then checked by flow cytometry, according to Cossarizza and Salvioli. ${ }^{18}$ Essentially, control and JAHA-treated MDA-MB231 cells, both attached and floating, harvested after 24 and $48 \mathrm{~h}$ from treatment, were stained with JC1 (final concentration, $2.5 \mu \mathrm{g} / \mathrm{mL}$ ) for $10 \mathrm{~min}$ at $37^{\circ} \mathrm{C}$, washed with phosphate-buffered saline (PBS), and analyzed for the green and orange fluorescence in a FACSCanto apparatus. Data were represented as dot plots using Flowing software v.1.6.0., which discriminate in the bottom quadrants the amount of cells that undergo loss of MMP.

Autophagy Assays. Quantification of Acidic Vesicular Organelles (AVOs). AVOs, which are a hallmark of autophagy, were quantified by flow cytometry as reported by Jiang et al. ${ }^{19}$ and Chen et $\mathrm{al}^{20}$ Essentially, control and JAHA-treated MDA-MB231 cells, both attached and floating, were harvested after 24 and $48 \mathrm{~h}$ of incubation and stained with acridine orange (final concentration, $100 \mu \mathrm{g} / \mathrm{mL}$ ) for $20 \mathrm{~min}$ in the dark and then analyzed in a FACSCanto flow cytometer. Data were represented as dot plots using Flowing software v.1.6.0., which discriminate cells with increased AVO accumulation in the top quadrants. In a parallel set of assays, as a negative control, untreated cells were incubated with bafilomycin A1 (Enzo Life Sciences), a 
macrolide antibiotic that inhibits the vacuolar type $\mathrm{H}^{+}$ATPase of $\mathrm{AVOs}^{20}$ at $200 \mathrm{nM}$ final concentration for $30 \mathrm{~min}$ before acridine orange staining.

Western Blot for LC3 and p62/Sequestosome 1 (p62/SQSTM1) Proteins. Electrophoretic analysis and immunoblots were performed according to Chiarelli et al. ${ }^{21}$ Essentially, control and JAHA-treated cell lysates obtained after 18,24 , and 48 h of culture were submitted to $8 \%$ SDS-PAGE and protein transfer onto Hybond-ECL nitrocellulose membranes (Amersham) using a semidry apparatus (Novablot, Pharmacia). Immunorevelation was performed with either of the two primary antibodies, that is, rabbit polyclonal anti-LC3 (L8918, Sigma, St. Louis, MO; 1:1000) or anti-p62/SQSTM1 (P0068, Sigma; 1:1000), and antiactin antibodies (A5060, Sigma; 1:500) as a loading control and secondary alkaline phosphatase-conjugated antirabbit IgG (Fc) antibody (S373B, Promega, Madison, WI), followed by incubation with 5-bromo-4-chloro-3'-indolyphosphate $p$-toluidine salt/nitro-blue tetrazolium chloride (BCIP/NBT) solution (Sigma). The intensities of the bands of interest, evaluated with ImageJ software, were normalized for those of actin. Data obtained by protein blot analysis are presented as means \pm standard errors of the mean (SEMs) of triplicate experiments.

Involvement of Autophagy Inhibition in the Cytotoxic Effect. JAHA-treated MDA-MB231 cells were incubated for $48 \mathrm{~h}$ with $1 \mathrm{nM}$ rapamycin (Santa Cruz Biotechnology, Santa Cruz, CA), an antibiotic that increases autophagosome and autolysosome formation, ${ }^{22}$ and the reversion of JAHA-triggered cytotoxic effect was monitored by an MTT assay, as previously reported. ${ }^{23}$ Control cells were tested in parallel.

\section{RESULTS}

In a first set of experiments, we checked the effect of dose- and time-dependent incubation with JAHA and homo-JAHA (Figure 1A) on MDA-MB 231 cell viability via MTT assay. As shown in Figure 1B, when cells were exposed for $72 \mathrm{~h}$ to JAHA, this compound caused death in a concentrationdependent manner with an $\mathrm{IC}_{50}=8.45 \mu \mathrm{M}$, and such concentration was chosen for all of the subsequent biochemical experiments. On the other hand, after $72 \mathrm{~h}$ of treatment, homoJAHA at the highest concentration used (i.e., $100 \mu \mathrm{M}$ ) reduced cell viability only to $63 \pm 1.8 \%$. The same concentration of either JAHA and homo-JAHA determined a decrease of cell viability to $83.9 \pm 5.1$ and $83.15 \pm 9.7 \%$ at $24 \mathrm{~h}$ of incubation and $66.4 \pm 6.7$ and $61.54 \pm 2.09 \%$, respectively. Phase-contrast microscopical observation of MDA-MB231 cells treated with 8.45 $\mu \mathrm{M}$ JAHA showed visible effects of cell death such as decreased cell number and the presence of rounded and floating cells, with respect to the firmly spread control cell preparations (Figure 1C).

Therefore, we performed a set of experiments aimed to get more detailed data on the molecular mechanism of JAHAinduced toxicity on MDA-MB231 cells. First, samples of control and JAHA-treated cells were submitted to annexin VFITC/propidium iodide (PI) staining and flow cytometric measurement to investigate the effect of JAHA on phosphatydilserine externalization, as a hallmark of induction of apoptosis, after short-term $(5 \mathrm{~h})$, and longer term (24 and $48 \mathrm{~h}$ ) treatments. As shown in Figure 2, JAHA treatment appeared to elicit a nonapoptotic cytotoxic effect as indicated by the increase of the percentage of necrotic cells in both top quadrants at $48 \mathrm{~h}$ of exposure and the almost total absence of apoptotic annexin ${ }^{+} / \mathrm{PI}^{-}$cells in the bottom right quadrant at all times of investigation.

Then, we wanted to check which kind of perturbation was induced by JAHA treatment on the MDA-MB231 cell cycle. To this purpose, cells treated with JAHA for 24 and $48 \mathrm{~h}$ were stained with PI and subjected to flow cytometric analysis of the

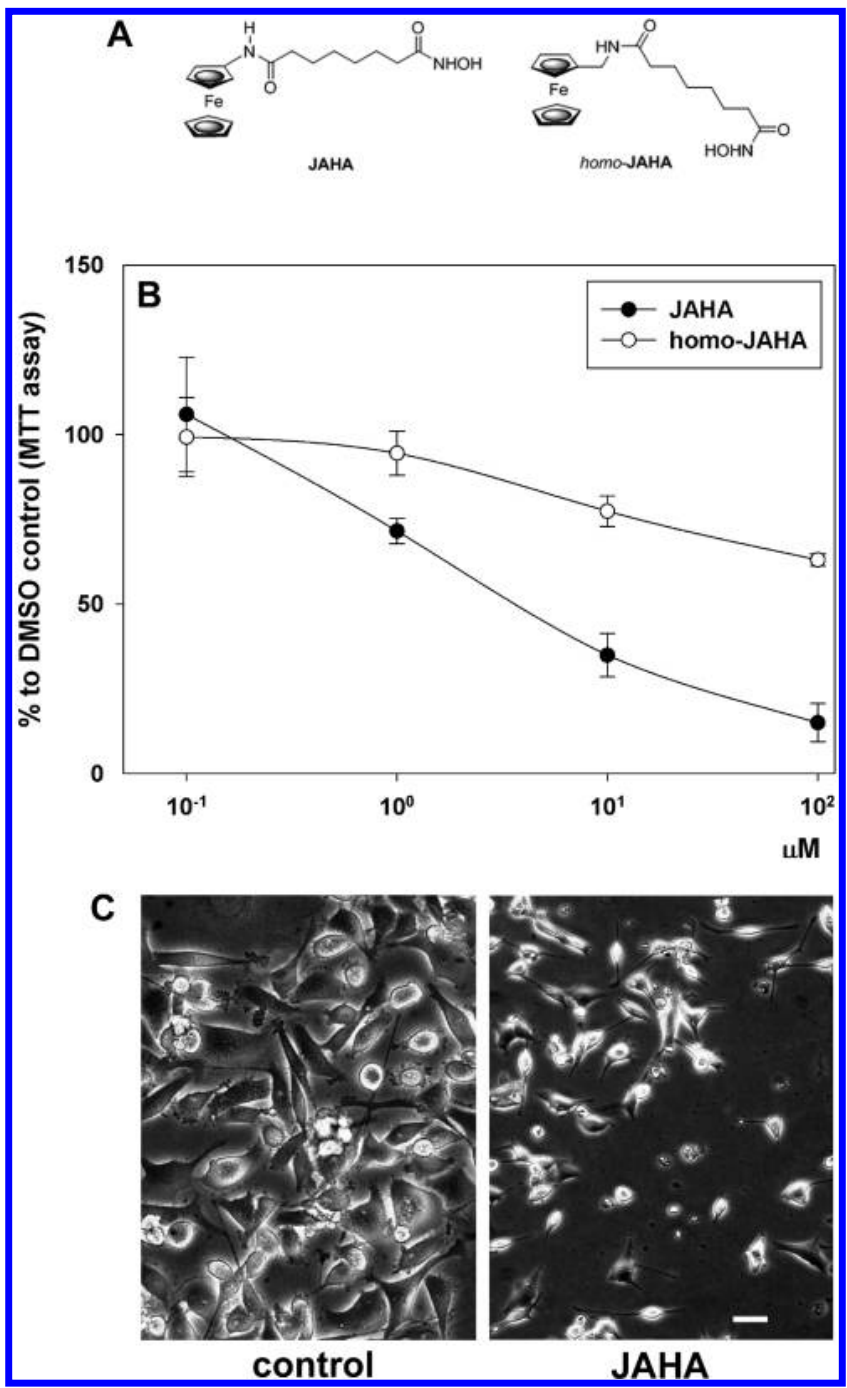

Figure 1. JAHAs and effect of $72 \mathrm{~h}$ of incubation on the viability of MDA-MB231 cells. (A) Structure of JAHA and homo-JAHA. (B) Dose-response curves for JAHA and homo-JAHA from triplicate MTT assays. The results are expressed as means \pm SEMs. (C) Representative phase-contrast micrographs of control and $8.45 \mu \mathrm{M}$ JAHA-treated cells showing changes in cell number and morphology after exposure to JAHA. Bar $=10 \mu \mathrm{M}$.

distribution of cell cycle phases. Figure 3 shows that, if compared to controls, exposure to JAHA was linked with a higher percentage of cells in the subG $\mathrm{G}_{0}$ fraction (66.22 and $47.20 \%$ vs 47.86 and $35.11 \%$, respectively, at 24 and $48 \mathrm{~h}$ ), consistent with an increase of damaged and fragmented cells due to compound toxicity more prominent at earlier times, a pronounced decrease of the $S$ phase fraction (6.68 and $8.28 \%$ vs 19.07 and $20.39 \%$, respectively, at 24 and $48 \mathrm{~h}$ ) and also of the $24 \mathrm{~h} \mathrm{G}_{0} / \mathrm{G}_{1}$ phase fraction ( 11.55 vs $21.17 \%$ ). Noteworthy, flow cytometric analysis also showed an increase of the $48 \mathrm{~h} \mathrm{G}_{2}$ phase fraction ( 15.53 vs $8.23 \%$ ), indicative of the acquisition of the inability to proceed through the $G_{2} / M$ transition upon prolonged incubation.

To detect variations of MMP after exposure to JAHA, we used the JC1 probe that is selectively uptaken into mitochondria, undergoing a fluorescence emission shift from green $(\sim 529 \mathrm{~nm})$ to red $(\sim 590 \mathrm{~nm})$ in the case of intact MMP, whereas in the case of mitochondrial depolarization, a decrease in the red/green fluorescence intensity ratio can be observed. ${ }^{17}$ 


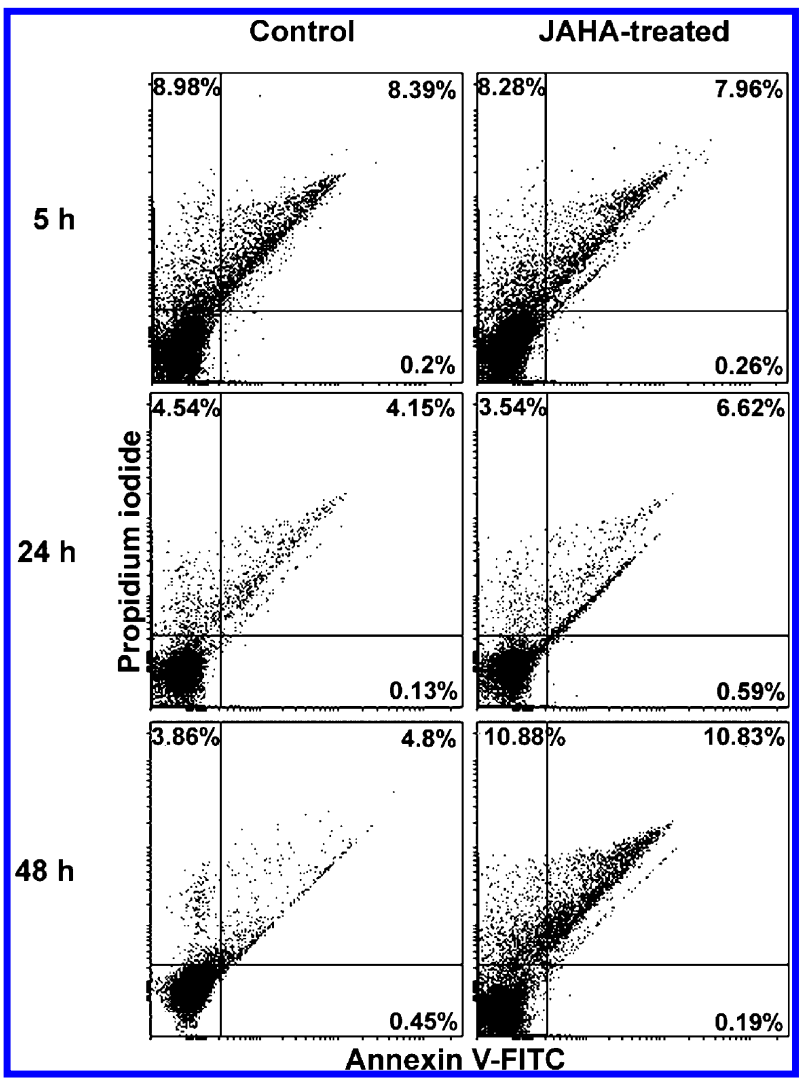

Figure 2. Flow cytometric analysis of control and JAHA-treated MDAMB231 cells stained with annexin V-FITC and PI for detection of phosphatydilserine externalization after 5,24 , and $48 \mathrm{~h}$ of exposure. The percentage indicated in the four quadrants in each frame refers to necrotic annexin ${ }^{-} / \mathrm{PI}^{+}$and annexin ${ }^{+} / \mathrm{PI}^{+}$cells (both top quadrants), apoptotic annexin ${ }^{+} / \mathrm{PI}^{-}$cells (bottom right quadrant), and normal annexin ${ }^{-} / \mathrm{PI}^{-}$cells (bottom left quadrant). Upon treatment, apoptotic cells never accounted for more than $0.59 \%$ of the cell population, whereas the percentage of necrotic cells showed an increase at longterm exposure.

As shown in Figure 4, flow cytometry analysis puts in evidence the time-dependent loss of the MMP in JAHA-treated cells, the percentage of low red-emitting cells (bottom quadrants) being about $9 \%$ after $24 \mathrm{~h}$ of exposure and increasing up to about $36 \%$ at $48 \mathrm{~h}$ of incubation, versus 2 and $5 \%$ of control cells, respectively. The JAHA ability to affect mitochondrial metabolism was also assessed through evaluation of ROS production using a commercial kit, which differentiates between total ROS and superoxide. As shown in Figure 5A, exposure to JAHA determined an early 3-fold increase of total intracellular ROS within $24 \mathrm{~h}$ of incubation; on the other hand, such upregulation of total ROS was not recorded at $48 \mathrm{~h}$ of treatment. In addition, although the superoxide-positive fraction of MDAMB231 cells treated with JAHA for $48 \mathrm{~h}$ was higher than that of controls ( 19.69 vs $4.05 \%$ ), the value was consistently less than half of that obtained with the pyocyanin-treated sample as positive control, thereby indicating that the observed upregulation of superoxide ion was not significant (data not shown). To check whether the observed ROS up-regulation could be involved, at least in part, in the onset of JAHAtriggered cytotoxicity, MDA-MB231 cells were coincubated with $8.45 \mu \mathrm{M}$ JAHA and the phenolic antioxidant BHT at increasing concentrations. The results shown in Figure $5 \mathrm{~B}$ demonstrate that the cotreatment was capable of reversing the

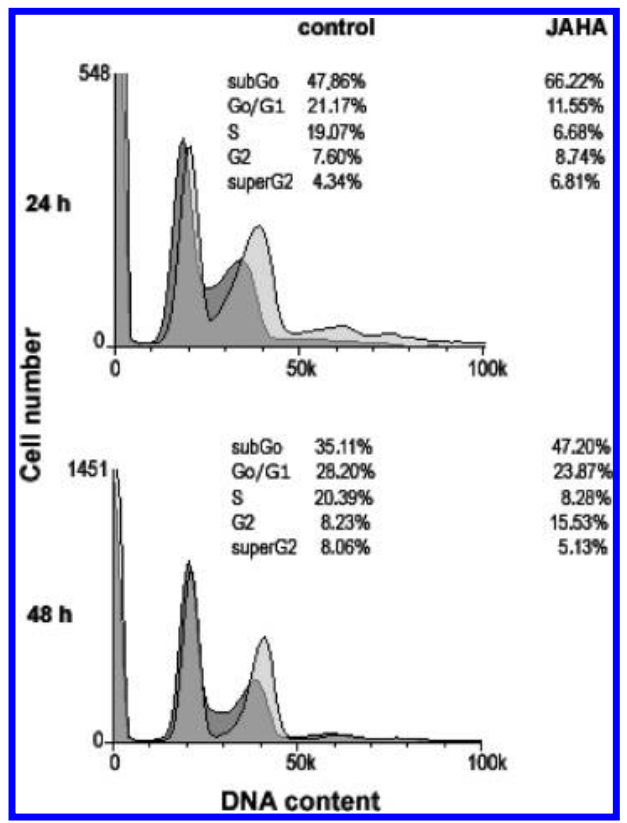

Figure 3. DNA profiles of control (darker in the background) and JAHA-treated (lighter superimposed) MDA-MB231 cells after 24 and $48 \mathrm{~h}$ of exposure. Cell cycle distribution is reported in the annexed tables for both cell samples.

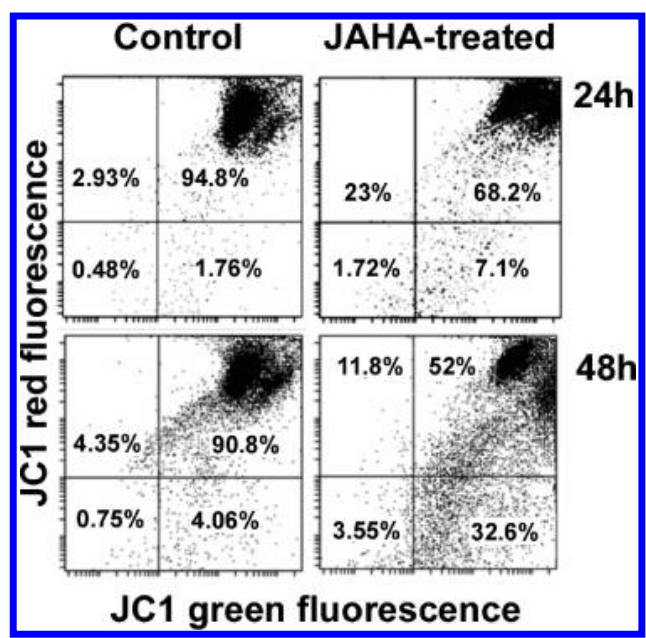

Figure 4. Analysis of MMP in control and JAHA-treated MDAMB231 cells. Flow cytometric analysis of control and JAHA-treated MDA-MB231 cells stained with JC1 after 24 and $48 \mathrm{~h}$ of exposure. The percentages indicated in the bottom quadrants in each frame refer to low red-emitting cells that underwent dissipation of MMP.

decrease in cell number recorded with JAHA-only treatment, with 25 and $50 \mu \mathrm{M}$ BHT concentrations, respectively, inducing a comparable and more pronounced cell growth than that of the untreated control.

Lastly, we checked whether JAHA determined a modification in the amount of autolysosomes, a hallmark of autophagy, through staining with acridine orange, a cell-permeable fluorescent dye that can be sequestered by acidic compartments after uptake and protonation. Figure 6 shows that JAHA-treated cells underwent a consistent reduction of AVO accumulation between 24 and $48 \mathrm{~h}$ of exposure and that such a decrease was comparable to that observed when cells were incubated with $200 \mathrm{nM}$ bafilomycin A1, a well-known inhibitor of autolysosome maturation, $^{24}$ as a negative control. Autophagy 


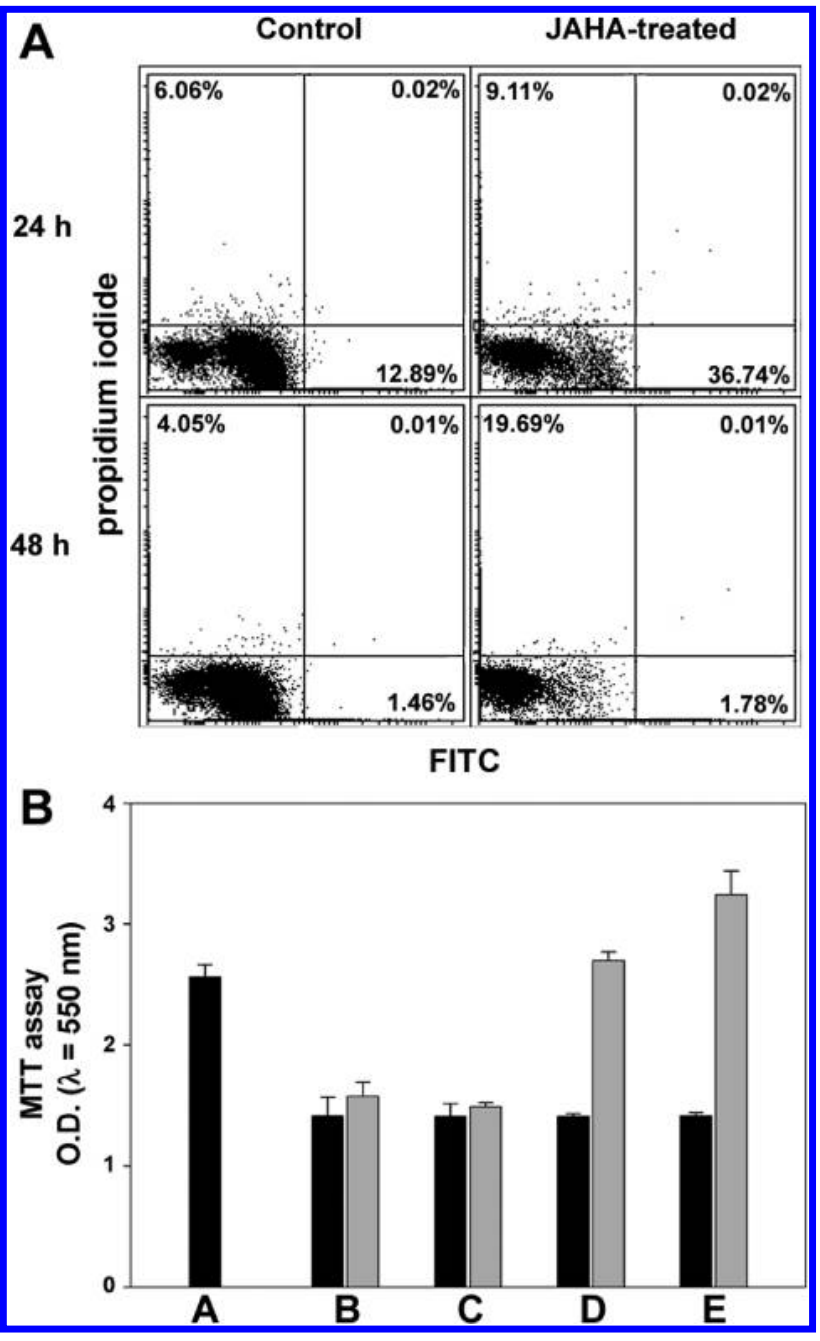

Figure 5. Effect of JAHA treatment on ROS production by MDAMB231 cells and its involvement in the cytotoxic effect. (A) Flow cytometric analysis of control and JAHA-treated MDA-MB231 cells stained with two color ROS detection reagents, after 24 and $48 \mathrm{~h}$ of exposure. The percentage indicated in the four quadrants in each frame refers to superoxide only overproducing cells (top left quadrant), total ROS overproducing cells (bottom right quadrant), and total ROS/superoxide overproducing cells (top right quadrant). Upon treatment, MDA-MB231 cells undergo a 3-fold up-regulation of total ROS within $24 \mathrm{~h}$ of exposure. The up-regulation of superoxide ion recorded at $48 \mathrm{~h}$ is not significant, the value being less than half of that of pyocyanin-treated sample, used as a positive control (data not shown). (B) MTT assay of control (A) and JAHA-treated (B-E, black bars) MDA-MB231 cells assayed in parallel with cells coincubated with $8.45 \mu \mathrm{M}$ JAHA and BHT at $5,10,25$, and $50 \mu \mathrm{M}$ concentrations (B$\mathrm{E}$, gray bars). The culture was grown for $24 \mathrm{~h}$, and the assay was made in triplicate. The results are expressed as means \pm SEMs. BHT at 25 and $50 \mu \mathrm{M}$ concentrations induced a reversal of the cytotoxic effect exerted by JAHA.

modulation by JAHA treatment was also monitored via molecular markers through immunoblot analysis. In particular, we checked (i) the conversion of microtubule-associated protein light chain 3 (LC3) from its cytosolic form (LC3-I) to its autophagosome-associated lipidated form (LC3-II), which differs for its lower molecular mass and can be discriminated through SDS-PAGE, ${ }^{25}$ and (ii) the changes in the accumulation levels of p62/SQSTM1, a receptor for ubiquitinated proteins that selectively delivers them into the autophagosome and is known to be a specific degradation substrate for autophagy. ${ }^{19}$ After the Western blot, the densitometry of the immunostained bands was performed with the ImageJ software. The immunoblot in Figure 7A shows that LC3-II was decreased in MDA-MB231 cells after $48 \mathrm{~h}$ of exposure to JAHA. In particular, Figure 7B,C reports the densitometric analyses referred to the LC3-II/LC3-I ratio and also to the LC3-II band only, as suggested by the advice of Mizishuma and Yoshimori. ${ }^{25}$ In both cases, although the difference between control and exposed cells was not evident at $24 \mathrm{~h}$, apart from a faint decrease of the band intensity ratio, the increase of the duration of incubation with JAHA up to $48 \mathrm{~h}$ resulted in a reduction of both the LC3-II/LC3-I ratio and the intensity of LC3-II band down to about 52 and $71 \%$, respectively, thus confirming the flow cytometric data. Further evidence of JAHA-induced autophagy down-regulation was also given by the immunoquantitation of p62/SQSTM1. In fact, as shown in Figure 7D, the p62/SQSTM1 amount in JAHA-treated cells normalized for that of actin increased by about $50 \%$ after $48 \mathrm{~h}$ of incubation, whereas it remained constant at $24 \mathrm{~h}$ of culture. Interestingly, when we evaluated the amount of LC3-I, LC3-II, and $\mathrm{p} 62 / \mathrm{SQSTM} 1$ after shorter term JAHA treatment, that is, at $18 \mathrm{~h}$ from cell plating, we found an increase of the LC3-II/ LC3-I ratio by about $62 \%$ and a reduction of p62/SQSTM1 down to about $20 \%$ (Figure 8), consistent with an early autophagy induction, which is then followed by an arrest of the autophagic flux over $24-48 \mathrm{~h}$ of treatment.

To check whether inhibition of the autophagic process could account, at least in part, for the observed JAHA-triggered lethal effect on MDA-MB231 cells, cells were coincubated with 8.45 $\mu \mathrm{M}$ JAHA and increasing concentrations of rapamycin (sirolimus), an antibiotic acting as autophagy promoter via inhibition of the mammalian target of rapamycin (mTOR), a serine-threonine kinase that plays a key role in regulating fundamental cellular activities, such as growth, proliferation, cytoskeletal architecture, transcription, and translation. ${ }^{26}$ When MDA-MB231 cells were coincubated with $8.45 \mu \mathrm{M}$ JAHA and $1 \mathrm{~nm}$ rapamycin, by analogy with BHT treatment, we found that also this cotreatment was capable of reversing the JAHAtriggered decrease in cell number (Figure 9), thereby revealing autophagy inhibition as a further aspect, supplementary to early ROS production, involved in the cytotoxic effect exerted by the HDACi under study on MDA-MB231 breast tumor cells.

\section{DISCUSSION}

Breast tumor is a widely spread neoplastic histotype accounting for about $20 \%$ of all cancers in women; about $15 \%$ of breast cancers are triple-negative, and the lack of receptors for estrogens, progesterone, and epidermal growth factor renders neoplastic cells highly aggressive and endowed with a higher malignant potential than other breast tumor subtypes. ${ }^{27}$ Because the pharmacological options for treating triple-negative tumors are limited, there has been great interest in testing novel drugs or analogues of pre-existing drugs counteracting triplenegative cell growth, which, on the other hand, necessitate a comprehensive biological characterization.

SAHA is an archetypal HDACi whose effect on triplenegative MDA-MB231 breast cancer cells, the model system used in the present study, has been previously tested. In fact, data from Huang and Pardee ${ }^{28}$ and $\mathrm{Xu}$ et al. ${ }^{29}$ demonstrated that SAHA affected cell cycle progression and induced polyploidy, the onset of apoptosis, and also the up-regulation of several proliferation-inhibiting and differentiation-promoting 


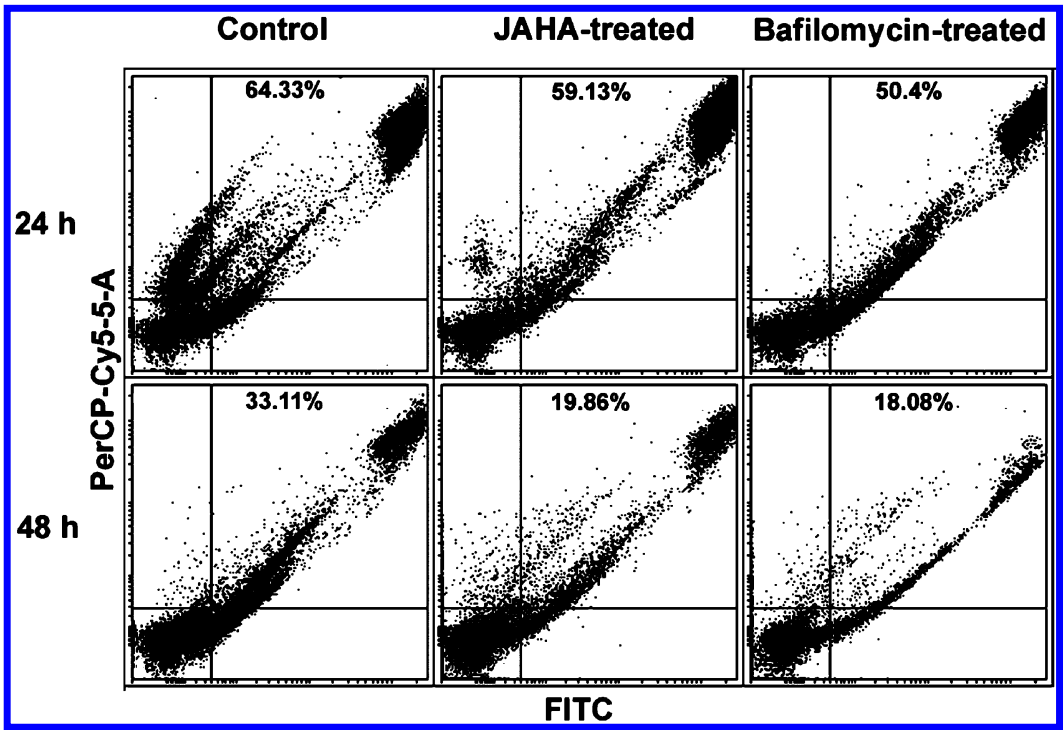

Figure 6. Effect of JAHA treatment on AVO accumulation in MDA-MB231 cells. Flow cytometric analysis of control, JAHA-, and bafilomycin A1treated MDA-MB231 cells stained with acridine orange, after 24 and $48 \mathrm{~h}$ of exposure. The percentage indicated refers to AVO-positive cells. JAHA treatment induced a consistent reduction of AVO accumulation between 24 and $48 \mathrm{~h}$ of exposure, comparable to that observed when cells were incubated with autophagy-inhibitor bafilomycin A1.

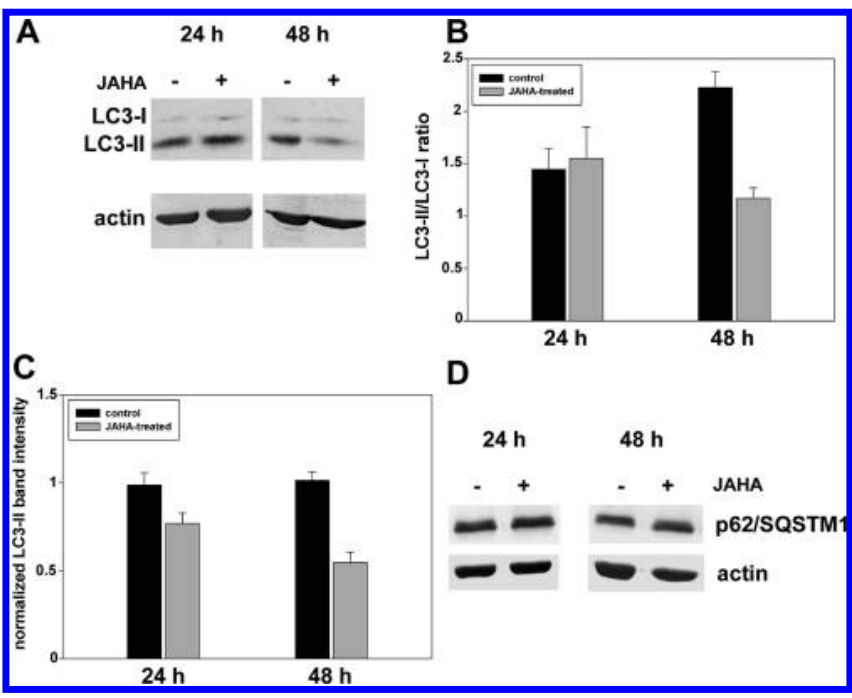

Figure 7. Effect of JAHA treatment for 24 and $48 \mathrm{~h}$ on autophagosome formation in MDA-MB231 cells. (A) SDS-PAGE and Western blotting for LC3-I and -II of whole-cell lysates of samples grown in control conditions or exposed to $8.45 \mu \mathrm{M}$ JAHA for 24 and $48 \mathrm{~h}$. $\beta$-Actin was used as a loading control. (B and C) Histograms showing the LC3-II/LC3-I ratio (B) and the normalized LC3-II band intensity (C) of control (black bars) and JAHA-treated (gray bars) MDA-MB231 cells after 24 and $48 \mathrm{~h}$ of culture. The results are expressed as means \pm SEMs of triplicate experiments. (D) SDS-PAGE and Western blotting for p62/SQSTM1 of whole-cell lysates of samples grown in control conditions or exposed to $8.45 \mu \mathrm{M} \mathrm{JAHA}$ for 24 and 48 h. $\beta$-Actin was used as the loading control.

genes, as assessed through differential display polymerase chain reaction (PCR) assays. The role of SAHA in triggering ROS production and dissipating MMP was reported in other cell model systems, for example, leukemia and colon carcinoma cells, although such events always have been associated to the promotion of apoptosis or autophagy by the HDACi. ${ }^{30-32}$ In addition, SAHA was proven to enhance autophagy also in other neoplastic cytotypes, such as hepatocellular carcinoma, chondrosarcoma, and glioblastoma cells. ${ }^{33-35}$

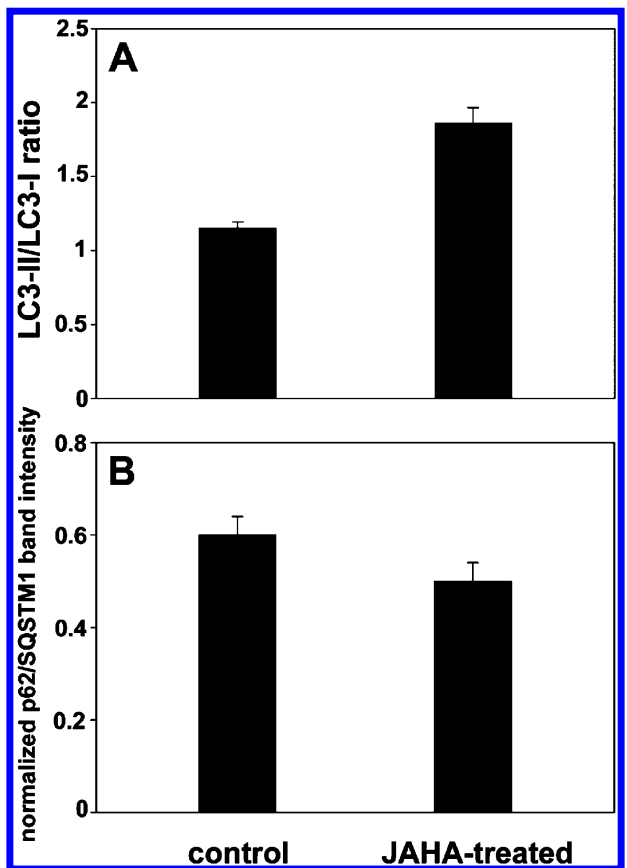

Figure 8. Effect of JAHA treatment for $18 \mathrm{~h}$ on autophagosome formation in MDA-MB231 cells. Histograms showing the LC3-II/ LC3-I ratio (A) and the normalized p62/SQSTM1 band intensity (B) of control and JAHA-treated cells.

In the present paper, we have tested the cytotoxic effect on triple-negative MDA-MB231 cells of two ferrocene-containing SAHA analogues, JAHA and homo-JAHA, already validated for their in vitro extensive inhibitory activity on class I HDACs, although to a lesser degree for HDAC8, and class IIb HDAC6. $^{12}$ The MTT viability assay demonstrated that if exposure to MDA-MB231 cells was prolonged to $72 \mathrm{~h}$, JAHA exhibited an $\mathrm{IC}_{50}$ of $8.45 \mu \mathrm{M}$, whereas homo-JAHA, which differs from JAHA for the presence of an additional methylene spacer, induced a maximum lowering of cell number down to about $63 \%$ only. Shorter term treatments of cells with JAHA, 


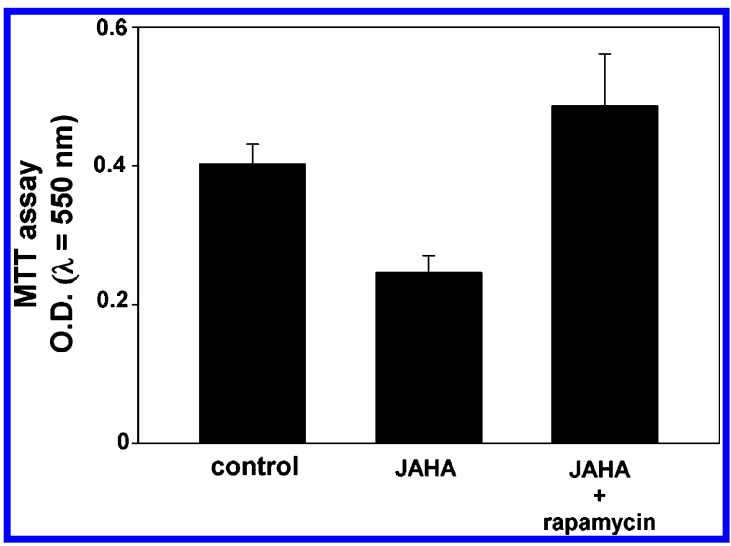

Figure 9. Effect of rapamycin cotreatment on JAHA-induced cytotoxicity on MDA-MB231 cells. MTT assay of control, JAHAtreated, and JAHA/rapamycin-cotreated MDA-MB231 cells. The culture was grown for $48 \mathrm{~h}$, and the assay was made in triplicate. The results are expressed as means \pm SEMs. Rapamycin at $1 \mathrm{nM}$ concentration induced a reversal of the cytotoxic effect exerted by JAHA.

that is, 24 and $48 \mathrm{~h}$, determined cell number reduction down to about only 84 and $66 \%$, respectively (data not shown).

The individuation of a cytotoxic effect exerted by JAHA on triple-negative cancer cells prompted a more detailed analysis of its mechanism of action, in particular focusing on cell cycle perturbation, and the possible onset of apoptosis and/or autophagy, coupled to ROS overproduction and/or MMP dissipation. Flow cytometric analysis of cell cycle phases produced similar data to those obtained on MCF-7 cells, ${ }^{12}$ which are shared with other HDACi (e.g., SAHA, trichostatin, apicidin, and psammaplin $\mathrm{A}$ ), that is, a perturbation of $\mathrm{S}$ phase entry and a delay at $G_{2} / M$ transition, ${ }^{24,36-39}$ although in our model system, we did not observe apoptotic cell death as with the other HDACi cited, this being a distinctive feature of JAHA.

The most intriguing results on JAHA mechanism of action regard its ability to induce early ROS production and subsequent MMP dissipation and autophagy inhibition, and the reversion of the cytotoxic effect obtained by cotreatment with either antioxidant or autophagy-promoter compound. It is well-known that ROS are generated by mitochondria where a multiplicity of producing sources is existent and where also ROS detoxifying systems are actively operating; therefore, the net intracellular amount of ROS derives from the imbalance of ROS production, removal, and extramitochondrial release. ${ }^{40}$ It can be hypothesized that JAHA treatment induces an oxidative stress due to mitochondrial defense failure, such as structural impairment and/or loss of matrix solutes (e.g., glutathione) through the permeability transition pores. On the other hand, initial ROS production may induce the onset of a "vicious cycle", ${ }^{40}$ that is, a further exacerbation of ROS formation due to the activation of additional ROS-generating sites within the mitochondria, which might be directly responsible for the following MMP collapse as already reported in other cell model systems ${ }^{41,42}$ and may be restrained in the presence of the antioxidant BHT.

Autophagy, that is, the formation of double membraneautophagosomes sequestering cytoplasmic material, which is digested due to subsequent fusion of the vesicles with the lysosomes, plays a complex role in breast cancer. In fact, some authors consider autophagy as a tumor suppressor mechanism leading to cell death, whereas other literature data strongly support the concept that this process is necessary to provide energy and basic elements allowing tumor cells to survive under conditions of metabolic stress, due to oxygen and nutrient shortage and rapid proliferative rate. ${ }^{22} \mathrm{~A}$ number of published papers suggest that the autophagy rate in MDA-MB231 cells is constitutively elevated, thereby playing a likely prosurvival role. For example, it is acknowledged that inhibition of autophagy sensitizes MDA-MB-231 cells to the lethal effects of chemical or physical agents (e.g., sulforaphane and capsaicin treatment or ionizing radiations) and that MDA-MB231 cells are resistant to rapamycin effects, probably due to their elevated phospholipase $\mathrm{D}$ activity that leads to the formation of phosphatidic acid, a lipid involved in autophagosome formation. ${ }^{23,43-47}$ The data here presented clearly indicate that JAHA can be included in the list of those molecules that trigger autophagy inhibition and deprive cells of such a protective mechanism, which can be restored upon rapamycin coincubation, addressing them to death. It is known that mitochondria play a central and complex role in autophagy. Recently, Okamoto ${ }^{48}$ reported that MMP plays a role in autophagy regulation and that defective mitochondria may impair the autophagic flux and vice versa through a protein kinase A-dependent mechanism. In addition, Kathiria et al. ${ }^{49}$ demonstrated that ROS up-regulation following mitochondrial membrane depolarization led to autophagy inhibition in human intestinal epithelial cells, and KarantzaWadsworth et al. ${ }^{50}$ proposed that in cases of defective autophagy, breast tumor cells lose the ability to adapt to metabolic stress, produce insufficient ATP, and accumulate damaged mitochondria with excessive ROS. In light of such evidence, we cannot exclude the occurrence of an interplay between the early ROS increase, MMP collapse, and autophagy down-regulation in JAHA-treated MDA-MB231 cells, although further studies will be required to confirm this hypothesis. Importantly, Rao et al. $^{51}$ reported the superior anticancer activity of cotreating triple-negative breast cancer cells and xenografts with the pan-HDACi panobinostat and the autophagy inhibitor chloroquine; therefore, the possible clinical utilization of the sole JAHA, an HDACi possessing an intrinsic autophagy-inhibiting activity, appears to be worth investigating. Noteworthy, the time-course study of JAHA effect on the control of the autophagic flux revealed a biphasic trend of the biological progress, whose molecular basis will deserve further and more detailed investigation.

In conclusion, two different SAHA analogues, JAHA and homo-JAHA, obtained by manipulation of SAHA aryl cap and assayed for their inhibitory action on purified HDACs, ${ }^{12}$ have been tested for their ability to restrain survival of a triplenegative breast cancer cell line in vitro. Of the two compounds, only JAHA exhibited a remarkable activity on MDA-MB231 cell viability and growth with an $\mathrm{IC}_{50}$ of $8.45 \mu \mathrm{M}$ at $72 \mathrm{~h}$. This indicates that the additional methylene spacer present in homoJAHA, which in the in tube assay exhibited a similar profile to that of JAHA for HDACs except HDAC6 and -8, affects its activity in a cellular environment and underlines the importance of a detailed biological characterization of promising bioinorganic probes for HDACis on specific cell model systems. Thus, JAHA, a ferrocene derivative of SAHA, provides a fascinating tool for studying the interactions of bioorganometallic agents in breast cancer. ${ }^{52,53}$ The results from biological assays of JAHA activity highlight the clear involvement of oxidative damage and autophagy inhibition in cytotoxicity, thus expanding the list of autophagy-regulating drugs and also giving 
an additional example of the role played by autophagy inhibition in breast cancer cell death.

Although caution must be exercised in extrapolation of in vitro results to the in vivo situation for which research on animals and human trials are needed, nevertheless, JAHA treatment possesses the potential for development as an agent for prevention and/or therapy of "aggressive" breast carcinoma, thus prompting us to get more insight into the molecular and biochemical pathways involved in JAHA antibreast cancer activity as well as into its possible genotoxic effects and modulatory functions in angiogenesis and cell invasion, which will be the object of forthcoming sets of experiments.

\section{AUTHOR INFORMATION}

\section{Corresponding Author}

*Tel: +3909123897405. Fax: +390916577210. E-mail: claudio. luparello@unipa.it.

\section{Funding}

This work was supported by a grant from the University of Palermo/Italy (R.S. ex60\%) to C.L.

\section{Notes}

The authors declare no competing financial interest.

\section{ACKNOWLEDGMENTS}

We thank Marco Vetrano and Marco Lapaglia for help in performing experiments during their laboratory internship.

\section{ABBREVIATIONS}

JAHA, Jay Amin hydroxamic acid; HDAC, histone deacetylase; HDACi, histone deacetylase inhibitor; SAHA, suberoylanilide hydroxamic acid; HER2, human epidermal growth factor receptor 2; MMP, mitochondrial membrane potential; FCS, fetal calf serum; MTT, 3-(4,5-dimethylthiazol-2-yl)-2,5-diphenyltetrazolium bromide; DMSO, dimethyl sulfoxide; $\mathrm{IC}_{50}$, half maximal inhibitory concentration; FITC, fluorescein isothiocyanate; ROS, reactive oxygen species; BHT, butylated hydroxytoluene; PBS, phosphate-buffered saline; AVO, acidic vesicular organelle; p62/SQSTM1, p62/sequestosome 1; $\mathrm{BCIP} / \mathrm{NBT}$, 5-bromo-4-chloro-3'-indolyphosphate $p$-toluidine salt/nitro-blue tetrazolium chloride; PI, propidium iodide; $\mathrm{PCR}$, polymerase chain reaction

\section{REFERENCES}

(1) Bell, O., Tiwari, V. K., Thomä, N. H., and Schübeler, D. (2011) Determinants and dynamics of genome accessibility. Nat. Rev. Genet. 12, 554-564.

(2) Majdzadeh, N., Morrison, B. E., and D'Mello, S. R. (2008) Class IIA HDACs in the regulation of neurodegeneration. Front. Biosci. 13, 1072-1082.

(3) Grabiec, A. M., Tak, P. P., and Reedquist, K. A. (2011) Function of histone deacetylase inhibitors in inflammation. Crit. Rev. Immunol. 31, 233-263.

(4) Hagelkruys, A., Sawicka, A., Rennmayr, M., and Seiser, C. (2011) The biology of HDAC in cancer: the nuclear and epigenetic components. Handb. Exp. Pharmacol. 206, 13-37.

(5) Manteuffel-Cymborowska, M. (1999) Nuclear receptors, their coactivators and modulation of transcription. Acta Biochim. Pol. 46, $77-89$.

(6) Green, C. D., and Han, J. D. (2011) Epigenetic regulation by nuclear receptors. Epigenomics 3, 59-72.

(7) Glozak, M. A., Sengupta, N., Zhang, X., and Seto, E. (2005) Acetylation and deacetylation of non-histone proteins. Gene 363, 1523.
(8) de Ruijter, A. J., van Gennip, A. H., Caron, H. N., Kemp, S., and van Kuilenburg, A. B. (2003) Histone deacetylases (HDACs): Characterization of the classical HDAC family. Biochem. J. 370 (Part 3), 737-749.

(9) Dickinson, M., Johnstone, R. W., and Prince, H. M. (2010) Histone deacetylase inhibitors: Potential targets responsible for their anti-cancer effect. Invest. New Drugs 28 (Suppl. 1), S3-S20.

(10) Kim, H. J., and Bae, S. C. (2011) Histone deacetylase inhibitors: Molecular mechanisms of action and clinical trials as anti-cancer drugs. Am. J. Transl. Res. 3, 166-179.

(11) Mann, B. S., Johnson, J. R., Cohen, M. H., Justice, R., and Pazdur, R. (2007) FDA approval summary: Vorinostat for treatment of advanced primary cutaneous T-cell lymphoma. Oncologist 12, 12471252 .

(12) Spencer, J., Amin, J., Wang, M., Packham, G., Alwi, S. S., Tizzard, G. J., Coles, S. J., Paranal, R. M., Bradner, J. E., and Heightman, T. D. (2011) Synthesis and biological evaluation of JAHAs: ferrocene-based histone deacetylase inhibitors. ACS Med. Chem. Lett. 2, 358-362.

(13) Kassam, F., Enright, K., Dent, R., Dranitsaris, G., Myers, J., Flynn, C., Fralick, M., Kumar, R., and Clemons, M. (2009) Survival outcomes for patients with metastatic triple-negative breast cancer: Implications for clinical practice and trial design. Clin. Breast Cancer 9, $29-33$.

(14) Gartel, A. L., Feliciano, C., and Tyner, A. L. (2003) A new method for determining the status of p53 in tumor cell lines of different origin. Oncol. Res. 13, 405-408.

(15) Luparello, C., David, F., Campisi, G., and Sirchia, R. (2003) T47-D cells and type V collagen: A model for the study of apoptotic gene expression by breast cancer cells. Biol. Chem. 384, 965-975.

(16) Luparello, C., Sirchia, R., and Lo Sasso, B. (2008) Midregion PTHrP regulates Rip1 and caspase expression in MDA-MB231 breast cancer cells. Breast Cancer Res. Treat. 111, 461-474.

(17) Cannino, G., Ferruggia, E., Luparello, C., and Rinaldi, A. M. (2008) Effects of cadmium chloride on some mitochondria-related activity and gene expression of human MDA-MB231 breast tumor cells. J. Inorg. Biochem. 102, 1668-1676.

(18) Cossarizza, A., and Salvioli, S. (2001) Flow cytometric analysis of mitochondrial membrane potential using JC-1. Curr. Protoc. Cytom., 14.1-14.7.

(19) Jiang, H., White, E. J., Conrad, C., Gomez-Manzano, C., and Fueyo, J. (2009) Autophagy pathways in glioblastoma. Methods Enzymol. 453, 273-286.

(20) Chen, Y., Azad, M. B., and Gibson, S. B. (2010) Methods for detecting autophagy and determining autophagy-induced cell death. Can. J. Physiol. Pharmacol. 88, 285-295.

(21) Chiarelli, R., Agnello, M., and Roccheri, M. C. (2011) Sea urchin embryos as a model system for studying autophagy induced by cadmium stress. Autophagy 7, 1028-1034.

(22) Armstrong, L. J., and Gorski, S. M. (2010) Breast cancer and autophagy. In Breast Cancer: Causes, Diagnosis and Treatment (Romero, M. E., and Dashek, L. M., Eds.) pp 53-88, Nova Science Publishers Inc., New York.

(23) Chang, S. B., Miron, P., Miron, A., and Iglehart, J. D. (2007) Rapamycin inhibits proliferation of estrogen-receptor-positive breast cancer cells. J. Surg. Res. 138, 37-44.

(24) Yamamoto, S., Tanaka, K., Sakimura, R., Okada, T., Nakamura, T., Li, Y., Takasaki, M., Nakabeppu, Y., and Iwamoto, Y. (2008) Suberoylanilide hydroxamic acid (SAHA) induces apoptosis or autophagy-associated cell death in chondrosarcoma cell lines. Anticancer Res. 28, 1585-1591.

(25) Mizushima, N., and Yoshimori, T. (2007) How to interpret LC3 immunoblotting. Autophagy 3, 542-545.

(26) Yang, Y. P., Liang, Z. Q., Gu, Z. L., and Qin, Z. H. (2005) Molecular mechanism and regulation of autophagy. Acta Pharmacol. Sin. 26, 1421-1434.

(27) Gluz, O., Liedtke, C., Gottschalk, N., Pusztai, L., Nitz, U., and Harbeck, N. (2009) Triple-negative breast cancer - current status and future directions. Ann. Oncol. 20, 1913-1927. 
(28) Huang, L., and Pardee, A. B. (2000) Suberoylanilide hydroxamic acid as a potential therapeutic agent for human breast cancer treatment. Mol. Med. 6, 849-866.

(29) Xu, W. S., Perez, G., Ngo, L., Gui, C. Y., and Marks, P. A. (2005) Induction of polyploidy by histone deacetylase inhibitor: a pathway for antitumor effects. Cancer Res. 65, 7832-7839.

(30) Dai, Y., Rahmani, M., Dent, P., and Grant, S. (2005) Blockade of histone deacetylase inhibitor-induced RelA/p65 acetylation and NFkappaB activation potentiates apoptosis in leukemia cells through a process mediated by oxidative damage, XIAP downregulation, and cJun N-terminal kinase 1 activation. Mol. Cell. Biol. 25, 5429-5444.

(31) Portanova, P., Russo, T., Pellerito, O., Calvaruso, G., Giuliano, M., Vento, R., and Tesoriere, G. (2008) The role of oxidative stress in apoptosis induced by the histone deacetylase inhibitor suberoylanilide hydroxamic acid in human colon adenocarcinoma HT-29 cells. Int. J. Oncol. 33, 325-331.

(32) Li, J., Liu, R., Lei, Y., Wang, K., Lau, Q. C., Xie, N., Zhou, S., Nie, C., Chen, L., Wei, Y., and Huang, C. (2010) Proteomic analysis revealed association of aberrant ROS signaling with suberoylanilide hydroxamic acid-induced autophagy in Jurkat T-leukemia cells. Autophagy 6, 711-724.

(33) Yamamoto, A., Tagawa, Y., Yoshimori, T., Moriyama, Y., Masaki, R., and Tashiro, Y. (1998) Bafilomycin Al prevents maturation of autophagic vacuoles by inhibiting fusion between autophagosomes and lysosomes in rat hepatoma cell line, H-4-II-E cells. Cell Struct. Funct. $23,33-42$.

(34) Liu, Y. L., Yang, P. M., Shun, C. T., Wu, M. S., Weng, J. R., and Chen, C. C. (2010) Autophagy potentiates the anti-cancer effects of the histone deacetylase inhibitors in hepatocellular carcinoma. Autophagy 6, 1057-1065.

(35) Gammoh, N., Lam, D., Puente, C., Ganley, I., Marks, P. A., and Jiang, X. (2010) Role of autophagy in histone deacetylase inhibitorinduced apoptotic and nonapoptotic cell death. Proc. Natl. Acad. Sci. U.S.A. 109, 6561-6565.

(36) Ueda, T., Takai, N., Nishida, M., Nasu, K., and Narahara, H. (2007) Apicidin, a novel histone deacetylase inhibitor, has profound anti-growth activity in human endometrial and ovarian cancer cells. Int. J. Mol. Med. 19, 301-308.

(37) Ahn, M. Y., Jung, J. H., Na, Y. J., and Kim, H. S. (2008) A natural histone deacetylase inhibitor, Psammaplin $\mathrm{A}$, induces cell cycle arrest and apoptosis in human endometrial cancer cells. Gynecol. Oncol. 108, 27-33.

(38) Noh, E. J., Lim, D. S., Jeong, G., and Lee, J. S. (2009) An HDAC inhibitor, trichostatin $\mathrm{A}$, induces a delay at $\mathrm{G} 2 / \mathrm{M}$ transition, slippage of spindle checkpoint, and cell death in a transcription-dependent manner. Biochem. Biophys. Res. Commun. 378, 326-331.

(39) Knutson, A. K., Welsh, J., Taylor, T., Roy, S., Wang, W. L., and Tenniswood, M. (2012) Comparative effects of histone deacetylase inhibitors on p53 target gene expression, cell cycle and apoptosis in MCF-7 breast cancer cells. Oncol. Rep. 27, 849-853.

(40) Andreyev, A. Y., Kushnareva, Y. E., and Starkov, A. A. (2005) Mitochondrial metabolism of reactive oxygen species. Biochemistry (Moscow) 70, 200-214.

(41) Abramov, A. Y., Scorziello, A., and Duchen, M. R. (2007) Three distinct mechanisms generate oxygen free radicals in neurons and contribute to cell death during anoxia and reoxygenation. J. Neurosci. 27, 1129-1138.

(42) Rogalska, A., Koceva-Chyła, A., and Jóźwiak, Z. (2008) Aclarubicin-induced ROS generation and collapse of mitochondrial membrane potential in human cancer cell lines. Chem.-Biol. Interact. $176,58-70$

(43) Chen, Y., Zheng, Y., and Foster, D. A. (2003) Phospholipase D confers rapamycin resistance in human breast cancer cells. Oncogene $22,3937-3942$

(44) Apel, A., Herr, I., Schwarz, H., Rodemann, H. P., and Mayer, A. (2008) Blocked autophagy sensitizes resistant carcinoma cells to radiation therapy. Cancer Res. 68, 1485-1494.

(45) Choi, C. H., Jung, Y. K., and Oh, S. H. (2010) Autophagy induction by capsaicin in malignant human breast cells is modulated by p38 and extracellular signal-regulated mitogen-activated protein kinases and retards cell death by suppressing endoplasmic reticulum stress-mediated apoptosis. Mol. Pharmacol. 78, 114-125.

(46) Kanematsu, S., Uehara, N., Miki, H., Yoshizawa, K., Kawanaka, A., Yuri, T., and Tsubura, A. (2010) Autophagy inhibition enhances sulforaphane-induced apoptosis in human breast cancer cells. Anticancer Res. 30, 3381-3390.

(47) Moreau, K., Ravikumar, B., Puri, C., and Rubinsztein, D. C. (2012) Arf6 promotes autophagosome formation via effects on phosphatidylinositol 4,5-bisphosphate and phospholipase D. J. Cell Biol. 196, 483-496.

(48) Okamoto, K. (2011) Mitochondria breathe for autophagy. EMBO J. 30, 2095-2096.

(49) Kathiria, A. S., Butcher, L. D., Feagins, L. A., Souza, R. F., Boland, C. R., and Theiss, A. L. (2012) Prohibitin 1 modulates mitochondrial stress-related autophagy in human colonic epithelial cells. PLoS One 7 (2), e31231 DOI: 10.1371/journal.pone.0031231.

(50) Karantza-Wadsworth, V., Patel, S., Kravchuk, O., Chen, G., Mathew, R., Jin, S., and White, E. (2007) Autophagy mitigates metabolic stress and genome damage in mammary tumorigenesis. Genes Dev. 21, 1621-1635.

(51) Rao, R., Balusu, R., Fiskus, W., Mudunuru, U., Venkannagari, S., Chauhan, L., Smith, J. E., Hembruff, S. L., Ha, K., Atadja, P., and Bhalla, K. N. (2012) Combination of pan-histone deacetylase inhibitor and autophagy inhibitor exerts superior efficacy against triple-negative human breast cancer cells. Mol. Cancer Ther. 11, 973-983.

(52) Osella, D., Homa Mahboobi, H., Colangelo, D., Cavigiolio, G., Vessieres, A., and Jaouen, G. (2005) FACS analysis of oxidative stress induced on tumour cells by SERMs. Inorg. Chim. Acta 358, 19931998.

(53) Spencer, J., Amin, J., Boddiboyena, R, Packham, G., Cavell, B. E., Syed Alwi, S. S., Paranal, R. M., Heightman, T. D., Wang, M., Marsden, B., Coxhead, P., Guille, M., Tizzard, G. J., Coles, S. J., and Bradner, J. E. (2012) Click JAHAs: Conformationally restricted ferrocene-based histone deacetylase inhibitors. Med. Chem. Commun. 3, $61-64$. 